\title{
In vitro maturation of goat oocytes selected using Brilliant cresyl blue staining
}

\author{
(iD) (iD ) (ID) \\ T. S. Arya ${ }^{\star 1}$, Amritha Aravind ${ }^{2}$, R. S. Abhilash ${ }^{3}$, C. Jayakumar ${ }^{4}$ and V. Babitha ${ }^{5}$ \\ Department of Animal Reproduction, Gynaecology and Obstetrics, \\ College of Veterinary and Animal Sciences, Mannuthy, Thrissur, Kerala, 680651 \\ Kerala Veterinary and Animal Sciences University, Kerala, India.
}

Citation: Arya, T. S., Amritha, A., Abhilash, R. S., Jayakumar, C. and Babitha, V. 2021. In vitro maturation of goat oocytes selected using Brilliant cresyl blue staining. J. Vet. Anim. Sci. 52(4): 405-408. DOI: https://doi.org/10.51966/jvas.2021.52.4.405-408

Received: 05.02.2021

Accepted: 09.03.2021

Published: 15.12.2021

\begin{abstract}
The present study was conducted to assess the developmental competence of goat oocytes selected using Brilliant cresyl blue (BCB) staining. Goat ovaries were collected from the slaughtered animals with unknown reproductive history. The oocytes retrieved by aspiration technique were selected based on morphology and subjected to BCB staining. Brilliant cresyl blue staining is based on the activity of glucose-6-phospahte dehydrogenase (G6PDH) enzyme synthesised by the oocytes. The cytoplasm remains blue in oocytes that have finished the growth phase $(B C B+)$ while the growing oocytes remain colourless (BCB-). The stained and unstained oocytes were subjected to in vitro maturation separately to assess cumulus cell expansion index and polar body extrusion. A total of 206 culture grade oocytes were subjected to study, out of which, $76.75 \pm 2.38$ per cent of oocytes showed positive to $B C B$ staining and $23.21 \pm 2.38$ per cent were negatively stained. Significantly higher maturation rate was observed in $B C B+(92.89 \pm$ $2.37 \%)$ oocytes than $B C B-(29.72 \pm 2.46 \%)$. The present study concluded that BCB staining can be used for selecting goat oocytes with good cytoplasmic maturation for further in vitro embryo production.
\end{abstract}

Key words: Goat oocytes, in vitro maturation, Brilliant cresyl blue

Running title: In vitro maturation of goat oocytes selected using Brilliant cresyl blue staining

Selection and identification of good quality oocytes is the critical step for in vitro embryo production which in turn ensures better in vitro maturation (IVM) and blastocyst formation (Egerszegi etal., 2010). Generally, the oocyte quality is determined by the morphological characters like number of cumulus cell layers and uniformity of the cytoplasm. A homogeneous population of oocytes with the same morphological characters differ in their developmental competence. Thus, BCB staining is proposed to assess the cytoplasmic competence which is predominantly responsible for oocyte competence.

1. MVSc Scholar

2. Assistant Professor

3. Assistant Professor

4. Assistant Professor and Head (i/c)

5. Assistant professor, Department of Veterinary Physiology

*Corresponding author: e-mail- aryats777@gmail.com Ph:8281671244

Copyright: (C) 2021 Arya et al. This is an open access article distributed under the terms of the Creative Commons Attribution 4.0 International License (http://creativecommons.org/licenses/by/4.0/), which permits unrestricted use, distribution, and reproduction in any medium, provided the original author and source are credited. 
Brilliant cresyl blue is a vital stain that allows to determine the intra cellular activity of the glucose-6-phospahate dehydrogenase (G6PDH) which is an enzyme synthesised in the cytoplasm of growing oocytes but remains low in the oocytes that have finished their growth phase. As the enzyme is active during growth phase, immature oocytes reduce $B C B$ to a colourless compound whereas, oocytes that have finished their growth phase show decreased activity of the G6PDH and their cytoplasm remain blue (Shabankareh et al., 2014). Studies in different farm species revealed that $B C B$ selected oocytes were having uniform distribution of mitochondria, more lipid content and also high glutathione level all indicating good developmental competence (Ghoneimy et al., 2017). The purpose of this study was to use $B C B$ staining as a selection tool to identify more competent goat oocytes having good cytoplasmic maturation for IVM and further processing for in vitro embryo production.

Goat ovaries transported to the laboratory within two hours of slaughter were washed several times in normal saline and removed excess tissue debris to reduce contamination. The visible surface follicles were aspirated and their contents were collected using a $10 \mathrm{~mL}$ disposable syringe attached to 20G needle. Oocyte collection medium (OCM) was used for retrieving cumulus oocyte complexes (COCs). Preliminary selection criteria for COCs were based on morphological characteristics like uniformity of cytoplasm and compactness of cumulus cells (Shioya et al., 1988). The COCs were graded according to the number of cumulus layers present around the oocyte (Koeman et al., 2003) as Grade-A ( $\geq 3$ cumulus layers), Grade-B (1-2 cumulus layers), Grade-C (denuded) and Grade-D (expanded cumulus). Grade A and B oocytes were selected for further in vitro studies.

The washed oocytes were subjected to BCB (B-5388-Sigma) staining by incubating at a concentration of $26 \mu \mathrm{M}$ at $37^{\circ} \mathrm{C}$ for $90 \mathrm{~min}$. The stained oocytes were washed three times in maturation medium and grouped as $\mathrm{BCB}+$ (blue coloured) and BCB- (colourless) depending on the blue colouration of cytoplasm (Fig.1). In vitro maturation was carried out separately for $\mathrm{BCB}+$ and BCB- oocytes. Microdroplets of $100 \mu \mathrm{L}$ maturation medium were prepared and 20 to 25 oocytes were transferred to each microdroplet under mineral oil. Incubation was carried out at $38.5^{\circ} \mathrm{C}$ in a humidified atmosphere under 5 per cent $\mathrm{CO}_{2}$ for $27 \mathrm{~h}$.

After IVM, the maturation status of oocytes was assessed based on the degree of cumulus cell expansion and extrusion of first polarbody in the perivitelline space. A total of 265 goat oocytes were collected from 353 follicles from 94 goat ovaries and classified based on the number of cumulus cell layer and uniformity of cytoplasm. Number for follicles obtained per ovary was $3.44 \pm 0.26$ and the number of COCs per ovary was $2.84 \pm 0.19$ with an oocyte recovery rate of 75.07 per cent. The maximum yield of COCs were obtained for grade A (48.43 $\pm 2.49 \%)$ followed by grade B $(27.23 \pm 2.22 \%)$. The yield of grade $C$ and $D$ were $12.28 \pm 2.43$ and $12.03 \pm 2.46$ per cent respectively. Since, only Grade A and B were considered as culture quality oocytes, they were selected for further in vitro studies. Out of 206 culture grade oocytes selected, $76.75 \pm 2.38$ per cent of oocytes showed positive to BCB staining and $29.72 \pm$ 2.46 per cent oocytes were negative to $B C B$ staining (Table 1). Maturation was assessed by cumulus cell expansion index following IVM. Most of the matured $\mathrm{BCB}+$ oocytes were having a cumulus expansion index of grade one and two, indicating good quality and BCB- oocytes were having more per cent of unexpanded oocytes of grade zero, indicating poor quality oocytes. The overall maturation rates of $\mathrm{BCB}+$ oocytes were found to be $92.89 \pm 2.37$ and $29.72 \pm 2.46$ per cent, respectively (Table 1 ). On statistical analysis, there was significant $(p<0.05)$ differences between the maturation rate of $\mathrm{BCB}+$ and $\mathrm{BCB}$ - oocytes.

Table 1. Yield and in vitro maturation rate of BCB selected goat oocytes

\begin{tabular}{|c|c|c|}
\hline $\begin{array}{c}\text { BCB } \\
\text { Staining }\end{array}$ & Yield (\%) & $\begin{array}{c}\text { In vitro } \\
\text { maturation rate } \\
\text { (\%) }\end{array}$ \\
\hline $\mathrm{BCB}+$ & $76.78 \pm 2.38^{\mathrm{a}}$ & $92.89 \pm 2.37^{\mathrm{a}}$ \\
\hline $\mathrm{BCB}-$ & $23.21 \pm 2.38^{\mathrm{b}}$ & $29.72 \pm 2.46^{\mathrm{b}}$ \\
\hline
\end{tabular}

** Significant at 0.01 level

Means having different letter as superscript differ significantly within a column 
Improvement in the oocyte screening can be achieved by the application of $B C B$ staining in different species. The G6PDH activity was associated with oocyte diameter, lipid content, mitochondrial distribution and high glutathione content and those oocytes were having good cytoplasmic maturation and higher developmental competence (Wu et al., 2007). The percentage of $B C B+$ oocytes were higher than BCB- oocytes as described by several authors (Mohammadi-Sangchehmeh et al., 2014 and Abazari-kia et al., 2014). Usually, the oocytes were selected on the basis of morphological criteria alone, but oocytes that appear similar in morphology might vary in their developmental capabilities post insemination. Hence, application of an additional selection tool to choose oocytes with good cytoplasmic maturation, such as BCB staining would improve the IVEP outcome. In the present study, a higher yield of $\mathrm{BCB}+$ oocytes than negative could be due to the fact that a preliminary selection was done based on morphology. Rodríguez-González et al., (2003) observed more maturation rate for $\mathrm{BCB}+$ oocytes than BCB-. Cumulus expansion is the indicator of the ability to provide energy, transport of the messenger molecules or mediate the effects of hormone during the period of maturation and presence of cumulus investment increases the fertilisation and embryo development rate compared with that of denuded oocytes (Tange et al., 2004).

Good maturation rate indicates the quality oocytes and therefore good developmental competence for further in vitro developments. The study revealed that the goat oocytes selected by $\mathrm{BCB}$ staining (BCB+ oocytes) had a higher per cent of maturation rate when compared to $\mathrm{BCB}$ - oocytes.

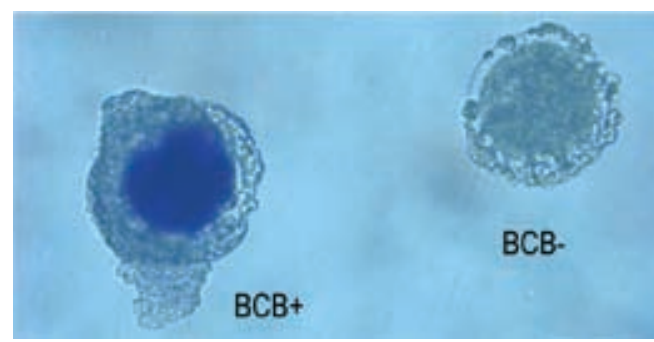

Fig. 1. Brilliant cresyl blue staining of goat oocytes (200X)

\section{Summary}

The oocytes selected by this method $(\mathrm{BCB}+)$ showed higher degree of cumulus cell expansion and maturation rate than BCBoocytes. Brilliant cresyl blue being a noninvasive stain, the selected oocytes can further be used for in vitro fertilization and embryo production. Hence, this staining method can be used as an effective tool for the selection of good quality goat oocytes for in vitro embryo production.

\section{Conflict of interest}

The authors declare that they have no conflict of interest.

\section{References}

Abazari-Kia, $\quad$ A.H., $\quad$ MohammadiSangcheshmeh, A., DehghaniMohammadabadi, M., JamshidiAdegani, F., Veshkini, A., Zhandi, M., Cinar, M.U. and Salehi, M. 2014. Intracellular glutathione content, developmental competence and expression of apoptosis-related genes associated with G6PDH-activity in goat oocyte. J. Assist. Reprod. Genet. 31: 313-321.

Egerszegi, I., Alm, H., Rátky, J., Heleil, B., Brüssow, K.P. and Torner, H. 2010. Meiotic progression, mitochondrial features and fertilisation characteristics of porcine oocytes with different G6PDH activities. Reprod. Fert. and Dev. 22(5): 830-838.

Ghoneimy, M. K., El-Naby, A.S., Youssef, Y. and El-Roos, A. 2017. Impact of using brilliant cresyl blue stain on oocyte and embryo selection. Egyptian J. of Vet. Sci. 48(1): 43-51.

Koeman, J., Keefer, C.L., Baldassare, H. and Downey, B.R. 2003. Developmental competence of prepubertal and adult goat oocytes cultured in semi defined media following laparoscopic recovery. Theriogenology. 60: 879-889. 
Mohammadi-Sangcheshmeh, A., Veshkini, A., Hajarizadeh, A., Jamshidi-Adegani, F., Zhandi, M., Abazari-kia, A.H., Cinar, M.U., Soleimani, M. and Gastal, E.L. 2014. Association of glucose-6phosphate dehydrogenase activity with oocyte cytoplasmic lipid content, developmental competence, and expression of candidate genes in a sheep model. J. Assist. Reprod. Genet. 31: 1089-1098.

Rodríguez-González, E., López-Bejar, M., Izquierdo, D. and Paramio, M.T. 2003. Developmental competence of prepubertal goat oocytes selected with brilliant cresyl blue and matured with cysteamine supplementation. Reprod. Nutr. Dev. 42: 79-187.

Shabankareh, K.H., Azimi, G. and Torki, M. 2014. Developmental competence of bovine oocytes selected based on follicle size and using the brilliant cresyl blue (BCB) test. Iran J Reprod Med. 12(11): 771-778.

Shioya,Y.,Kuwayama, M., Fukushima,M., Iwasaki, S. and Handa, A. 1998. In vitro fertilization and cleavage capacity of bovine follicular oocytes classified by cumulus cells and matured in vitro. Theriogenology. 30: 489-496.

Tange, T.O., Nott, A. and Moore, M.J. 2004. The ever-increasing complexities of the exon junction complex. Curr. opinion in cell biol. 16: 279-284.

Wu, Y.G., Liu, Y., Zhou, P., Lan, G.C., Han, D., Miao, D.Q. and Tan, J.H., 2007. Selection of oocytes for in vitro maturation by brilliant cresyl blue staining: a study using the mouse model. Cell Res. 17: 22-731. 